Geometry 85 Topology

Volume 7 (2003) 641-643

Published: 22 October 2003

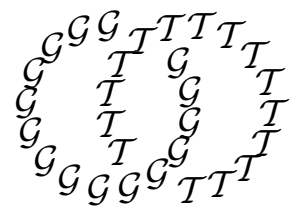

\title{
Splitting the concordance group of algebraically slice knots
}

\author{
Charles Livingston \\ Department of Mathematics, Indiana University \\ Bloomington, IN 47405, USA \\ Email: livingst@indiana.edu
}

\begin{abstract}
As a corollary of work of Ozsváth and Szabó [8], it is shown that the classical concordance group of algebraically slice knots has an infinite cyclic summand and in particular is not a divisible group.
\end{abstract}

\section{AMS Classification numbers Primary: 57M25}

Secondary: 57Q60

Keywords: Knot concordance, algebraically slice

Proposed: Robion Kirby

Seconded: Tomasz Mrowka, Cameron Gordon

(C) Geometry $\& \mathcal{G}$ Topology $\mathcal{P}$ ublications
Received: 1 June 2003

Accepted: 21 September 2003 
Let $\mathcal{A}$ denote the concordance group of algebraically slice knots, the kernel of Levine's homomorphism $\phi: \mathcal{C} \rightarrow \mathcal{G}$, where $\mathcal{C}$ is the classical knot concordance group and $\mathcal{G}$ is Levine's algebraic concordance group [6]. Little is known about the algebraic structure of $\mathcal{A}$ : it is countable and abelian, Casson and Gordon [2] proved that $\mathcal{A}$ is nontrivial, Jiang [5] showed it contains a subgroup isomorphic to $\mathbf{Z}^{\infty}$, and the author [7] proved that it contains a subgroup isomorphic to $\mathbf{Z}_{2}^{\infty}$. We add the following theorem, a quick corollary of recent work of Ozsváth and Szabó [8].

Theorem 1 The group $\mathcal{A}$ contains a summand isomorphic to $\mathbf{Z}$ and in particular $\mathcal{A}$ is not divisible.

Proof In [8] a homomorphism $\tau: \mathcal{C} \rightarrow \mathbf{Z}$ is constructed. We prove that $\tau$ is nontrivial on $\mathcal{A}$. The theorem follows since, because $\operatorname{Im}(\tau)$ is free, there is the induced splitting, $\mathcal{A} \cong \operatorname{Im}(\tau) \oplus \operatorname{Ker}(\tau)$. No element representing a generator of $\operatorname{Im}(\tau)$ is divisible.

According to [8], $|\tau(K)| \leq g_{4}(K)$, where $g_{4}$ is the 4-ball genus of a knot, and there is the example of the $(4,5)$-torus knot $T$ for which $\tau(T)=6$. We will show that there is a knot $T^{*}$ algebraically concordant to $T$ with $g_{4}\left(T^{*}\right)<6$. Hence, $T \#-T^{*}$ is an algebraically slice knot with nontrivial $\tau$, as desired.

Recall that $T$ is a fibered knot with fiber $F$ of genus $(4-1)(5-1) / 2=6$. Let $V$ be the $12 \times 12$ Seifert matrix for $T$ with respect to some basis for $H_{1}(F)$. The quadratic form $q(x)=x V x^{t}$ on $\mathbf{Z}^{12}$ is equal to the form given by $\left(V+V^{t}\right) / 2$. Using [3] the signature of this symmetric bilinear form can be computed to be 8 , so $q$ is indefinite, and thus by Meyer's theorem [4] there is a nontrivial primitive element $z$ with $q(z)=0$. Since $z$ is primitive, it is a member of a symplectic basis for $H_{1}(F)$. Let $V^{*}$ be the Seifert matrix for $T$ with respect to that basis. The canonical construction of a Seifert surface with Seifert matrix $V^{*}$ ([9], or see [1]) yields a surface $F^{*}$ such that $z$ is represented by a simple closed curve on $F^{*}$ that is unknotted in $S^{3}$. Hence, $F^{*}$ can be surgered in the 4 -ball to show that its boundary $T^{*}$ satisfies $g_{4}\left(T^{*}\right)<6$. Since $T^{*}$ and $T$ have the same Seifert form, they are algebraically concordant.

Addendum An alternative proof of Theorem 1 follows from the construction of knots with trivial Alexander polynomial for which $\tau$ is nontrivial, to appear in a forthcoming paper. 


\section{References}

[1] G Burde, H Zieschang, Knots, de Gruyter Studies in Mathematics, 5, Walter de Gruyter \& Co., Berlin (1985)

[2] A Casson, C McA Gordon, Cobordism of classical knots, from: "A la recherche de la Topologie perdue", (Guillou and Marin, editors), Progress in Mathematics, Volume 62 (1986), originally published as an Orsay Preprint (1975)

[3] C McA Gordon, R Litherland, K Murasugi, Signatures of covering links, Canad. J. Math. 33 (1981) 381-394

[4] D Husemoller, J Milnor, Symmetric Bilinear Forms. Ergebnisse der Mathematik und ihrer Grenzgebiete, Band 73, Springer-Verlag, New York-Heidelberg (1973)

[5] B Jiang, A simple proof that the concordance group of algebraically slice knots is infinitely generated, Proc. Amer. Math. Soc. 83 (1981) 189-192

[6] J Levine Knot cobordism groups in codimension two, Comment. Math. Helv. 44 (1969) 229-244

[7] C Livingston, Order 2 algebraically slice knots, from Proceedings of the Kirbyfest (Berkeley, CA, 1998) 335-342, Geom. Topol. Monogr., 2, Geom. Topol. Publ., Coventry (1999)

[8] P Ozsváth, Z Szabó, Knot Floer homology and the four-ball genus, Geometry and Topology 7 (2003) 615-639, arXiv:math.GT/0301149

[9] H Seifert, Über das Geschlecht von Knoten, Math. Ann. 110 (1934) 571-592 